\title{
Role of the gold film nanostructure on the nanomechanical response of microcantilever sensors
}

\author{
J. Mertens, M. Calleja, D. Ramos, A. Tarýn, and J. Tamayo ${ }^{\text {a) }}$ \\ BioNanoMechanics Laboratory, National Center for Microelectronics, IMM-CNM (CSIC), \\ Isaac Newton 8 (PTM), Tres Cantos, 28760 Madrid, Spain
}

(Received 23 May 2006; accepted 1 December 2006; published online 5 February 2007)

\begin{abstract}
In this study, we have determined the relationship between the nanostructure of the gold film deposited on microcantilevers and the sensitivity and reproducibility of their static response to molecular adsorption. In order to tune the properties of the gold film, gold was deposited at different rates and thicknesses. The cantilever response to molecular adsorption was characterized by exposure of the cantilevers to mercaptohexanol in water. The morphology of the gold surface was characterized by atomic force microscopy, and the residual stress induced in the cantilevers was characterized by a profilometry technique based on the optical beam deflection method. We have found that the discontinuous morphology of the gold film for small thicknesses and low deposition rates gives rise to large values of residual tensile stress due to the formation of grain boundaries at the expense of strain energy. These cantilevers exhibit the highest sensitivity and reproducibility to molecular adsorption. However, larger thicknesses and higher deposition rates produce the coalescence of gold nanoislands via atom diffusion. This is characterized by a large relative decrease (increase) of the tensile (compressive) stress. These cantilevers exhibit small sensitivity and low reproducibility to molecular adsorption. We conclude that the control of the gold coating process is critical for the reliability of the measurements with nanomechanical sensors. (C) 2007 American Institute of Physics. [DOI: 10.1063/1.2434011]
\end{abstract}

\section{INTRODUCTION}

Microcantilever sensors are attracting a growing interest due to their capabilities for high sensitivity, label-free detection, and small sample consumption. ${ }^{1,2}$ These properties are inherent to the nature of the nanomechanical response and the tiny size of the microcantilevers, with areas of the order of $1000 \mu \mathrm{m}^{2}$. In addition, these sensors are scalable as the microfabrication techniques allow routine fabrication of arrays of tens of cantilevers. Thus, this technology can respond to the need for platforms for complex chemical and biological analysis. The basic principle of these devices is the measurement of the nanometer-scale variation of the equilibrium position (static mode) and vibration (dynamic mode) of the cantilever due to molecular adsorption on the cantilever. Measurements in liquids are usually performed in the static mode due to the low quality factor of cantilevers in liquids that largely decreases the sensitivity of the dynamic mode. The mechanism responsible for the static signal (cantilever bending) is the buildup of differential surface stress when molecular adsorption preferentially occurs on one side of the cantilever. The differential surface stress between opposite surfaces is energetically relaxed by unequal expansion or contraction of both surfaces, resulting in a cantilever bending. In an approximate picture, the cantilever bends with a uniform curvature radius given by Stoney's equation, ${ }^{3}$

\footnotetext{
${ }^{a)}$ Author to whom correspondence should be addressed; electronic mail: jtamayo@imm.cnm.csic.es
}

$$
\frac{1}{R}=6 \frac{1-v}{E T^{2}}\left(\Delta \sigma_{t}-\Delta \sigma_{b}\right),
$$

where $R$ is the curvature radius of the thin plate, $\Delta \sigma_{t}-\Delta \sigma_{b}$ is the differential surface stress change between opposite sides (top and bottom) of the thin plate, $T$ is the thickness of the plate, and $E$ and $v$ are Young's modulus and the Poisson ratio, respectively.

The surface stress relates the energy change produced when a surface is elastically stretched to the resulting strain. In clean surfaces, the origin of the surface stress arises from the interactions between neighboring surface atoms and of those with the environment. A fundamental mechanism of surface stress is the charge redistribution as a consequence of the solid truncation at the surface. ${ }^{4,5}$ This mechanism can simply be understood as follows. In the bulk, atoms are positively charged and are bound by sharing either localized or delocalized electrons, whereas at the surface, electrons react to the missing atoms and bonded charge. Thus, the electronic charge is redistributed, flowing into the space between the surface atoms. The increase of charge density in the space between the surface atoms makes the elastic compression energetically favorable, indicating that the surface stress is positive, usually referred to as tensile surface stress. This behavior occurs in most of the clean metals, in which the surface stress is not strong enough to drive the surface into reconstruction. In the opposite case, when the energy of the surface is minimized during positive elastic strain, the surface stress is referred to as compressive. Adsorption of molecules on the surface highly modifies the surface stress since it produces a charge redistribution between the surface and 
the adsorbates. ${ }^{4,5}$ In addition to the charge transfer mechanism, the lateral interaction between adsorbed molecules plays an important role in the adsorption-driven surface stress, particularly at high coverages.

Differential molecular adsorption between opposite cantilever sides is usually accomplished by previous deposition of a thin gold film on one side of the cantilever. The gold surface can be selectively functionalized by adsorption of thiol self-assembled monolayers. Surface stress based sensors using gold-coated cantilevers have been used for highly sensitive and selective detection of biomolecules such as proteins and DNA. ${ }^{6-11}$ However, the reproducibility of these studies has been poor between different laboratories due to differences in instrumentation and surface chemistry. In particular, small attention has been paid to the role of the gold film in the cantilever response.

The properties of the gold coating can significantly influence the cantilever response to molecular adsorption. Firstly, it produces significant residual stress that may affect the surface stress induced by subsequent molecular adsorption. A major source of residual stress is the formation of grain boundaries during the coalescence of individually nucleated clusters. ${ }^{12-14}$ Secondly, it has been demonstrated that the nanostructure of the gold surface has important effects on the cantilever response due to an increased number of available binding sites and the confinement of the molecules in nanocavities. ${ }^{15}$ In addition, both the kinetics and arrangement of the molecules on the surface are strongly influenced by the surface structure of the underlying gold. ${ }^{16}$

The aim of this work is to study the relationship between the gold film nanostructure, the residual surface stress, and the microcantilever response to molecular adsorption. For this purpose, we have deposited gold films on the cantilever by physical vapor deposition in a variety of conditions that produce different morphological properties and stress in the gold layer. ${ }^{17}$ The growth conditions examined here were the deposition rate $(0.02,0.2$, and $0.3 \mathrm{~nm} / \mathrm{s})$ for a gold film thickness of $60 \mathrm{~nm}$ and the film thickness, from 10 to $80 \mathrm{~nm}$, at a deposition rate of $0.02 \mathrm{~nm} / \mathrm{s}$. The surface morphology at nanometer scale was characterized ex situ by atomic force microscopy (AFM). The residual stress of the cantilevers was characterized by a profilometry technique based on the combination of the optical beam deflection technique and the scanning of the incident laser beam. ${ }^{18,19}$ The effect of the gold film nanostructure and induced residual stress on the cantilever response to molecular adsorption was characterized by monitoring in situ the cantilever bending during the adsorption of the alkylthiol mercaptohexanol $(\mathrm{MCH})$ in water.

\section{MATERIALS AND METHODS}

\section{A. Gold film preparation and characterization}

We have used commercially available arrays of five silicon cantilevers spaced $250 \mu \mathrm{m}$ (Mikromasch). The cantilevers were $400 \mu \mathrm{m}$ long, $100 \mu \mathrm{m}$ wide, and $1.0 \mu \mathrm{m}$ thick. The gold layer on the cantilevers was deposited by thermal evaporation. In order to obtain different nanostructures and residual stress for the gold film, gold was deposited at dif- ferent rates $(0.02,0.2$, and $0.3 \mathrm{~nm} / \mathrm{s})$ and with different thicknesses from 10 to $80 \mathrm{~nm}$. The growth rate was controlled within $1 \%$ accuracy by means of a quartz crystal microbalance. An intermediate $2 \mathrm{~nm}$ thick chromium layer was deposited at $0.02 \mathrm{~nm} / \mathrm{s}$ to enhance the adhesion between the gold layer and the cantilever. The surface topography of gold films was characterized by AFM (Nanoscope III, Veeco), in tapping mode at room temperature and humidity. The surface roughness was determined by calculating the root mean square $(\mathrm{rms})$ of the height images for areas of $1 \times 1 \mu \mathrm{m}^{2}$.

\section{B. Measurement of the surface stress}

In order to characterize the surface stress induced on the cantilevers, we have developed an optical technique for measuring the profile of several cantilevers belonging to the same array. ${ }^{19}$ The technique is based on the automated twodimensional scanning of a laser beam and the measurement of the deflection of the reflected laser beam by a linear position sensitive photodetector (SiTek). The experimental setup consists of a $3 \mathrm{~mW}$ red laser diode (Edmund Optics) that is mounted on two perpendicular linear voice coil actuators. The voice coil actuators allow fine positioning of the laser beam with an accuracy of $100 \mathrm{~nm}$ over a range of several millimeters without hysteresis. The laser beam scanning can be performed at velocities of up to $50 \mathrm{~mm} / \mathrm{s}$. The laser beam size on the cantilever is of about $5-10 \mu \mathrm{m}$ by using a convergent lens. In a first scan, the laser beam sequentially illuminates the cantilevers. Maxima of photocurrent are obtained in the photodetector at the laser beam positions where each cantilever is illuminated. ${ }^{18}$ Once the cantilevers are located in the first scan, a second scan along each cantilever is performed to obtain the profile. The vertical coordinate of the reflected laser beam on the photodetector is proportional to the local slope at the position where the laser beam is illuminating the cantilever. By numerically integrating the measurement of the local slope along the cantilever, the cantilever profile is obtained. In the experiments, the scanning velocity along the cantilever was set to $0.2 \mathrm{~mm} / \mathrm{s}$.

\section{RESULTS}

\section{A. Morphology of the gold film}

Atomic force microscopy was used to characterize the surface topography of the different gold films deposited on the cantilevers.

Figure 1 shows topography images of three gold films with a thickness of $60 \mathrm{~nm}$ deposited at three deposition rates, $0.02,0.2$, and $0.3 \mathrm{~nm} / \mathrm{s}$. The surface of the gold film deposited at a rate of $0.02 \mathrm{~nm} / \mathrm{s}$ exhibits well-defined grains with a size of 50-70 $\mathrm{nm}$ and the formation of grain boundaries [Fig. 1(a)]. ${ }^{20}$ The surface roughness (root mean square) is $1.6 \mathrm{~nm}$. For a deposition rate of $0.2 \mathrm{~nm} / \mathrm{s}$, the grains exhibit a less uniform size distribution that ranges from 30 to $70 \mathrm{~nm}$ [Fig. 1(b)]. A higher number of grain boundaries were observed, which are more blurred, indicating an initial stage of coalescence. This is reflected in a decrease of the surface roughness to $1.2 \mathrm{~nm}$. When the deposition rate is further increased to $0.3 \mathrm{~nm} / \mathrm{s}$, the grains are $60-80 \mathrm{~nm}$ in diameter and show an elongated shape. Some grains are similar to 


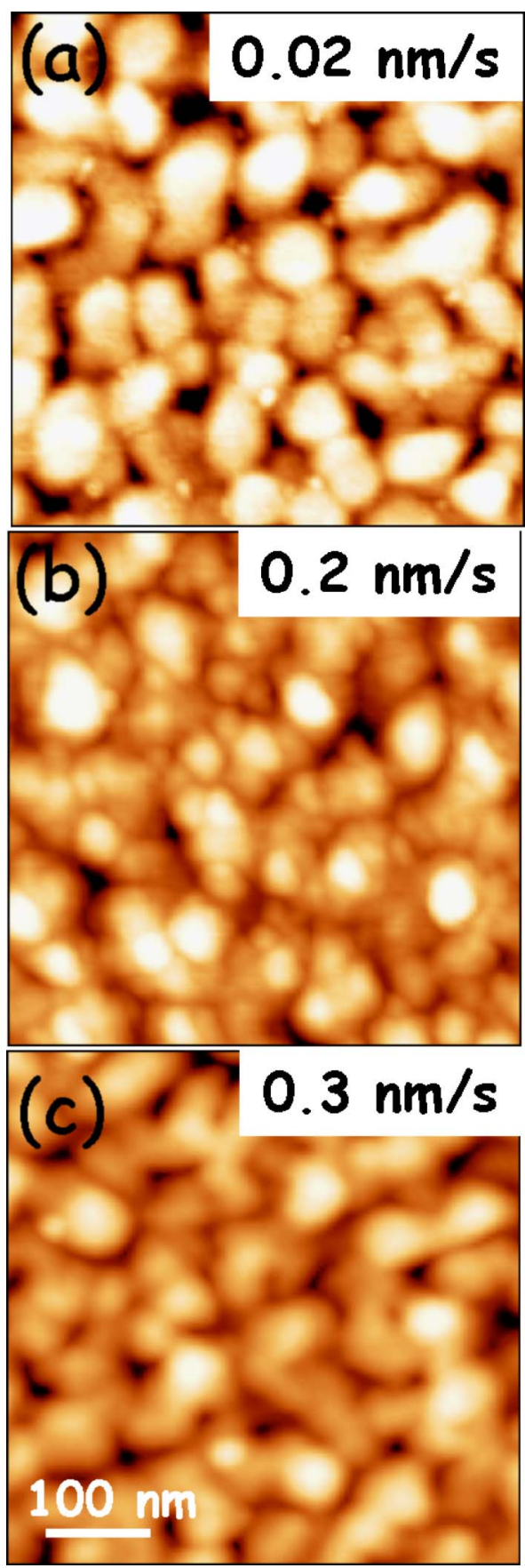

FIG. 1. (Color online) Atomic force microscopy topography images $(0.5$ $\times 0.5 \mu \mathrm{m}^{2}$ ) of $60 \mathrm{~nm}$ thick gold films deposited on the silicon cantilevers at (a) $0.02 \mathrm{~nm} / \mathrm{s}$, (b) $0.2 \mathrm{~nm} / \mathrm{s}$, and (c) $0.3 \mathrm{~nm} / \mathrm{s}$. An intermediate $2 \mathrm{~nm}$ thick chromium layer was deposited at $0.02 \mathrm{~nm} / \mathrm{s}$ to enhance the adhesion between the gold layer and the cantilever.

liquid droplets joined together through capillarylike necks [Fig. 1(c)]. The roughness slightly decreases to $1.1 \mathrm{~nm}$. This structure indicates a high degree of coalescence driven by diffusion of gold atoms. It has been demonstrated that a ductile material such as gold can be subjected to coalescence or "cold welding" far below the melting temperature. This is related to the high proportion of surface atoms, which are weakly bound and less constrained. ${ }^{21-23}$

The morphology of the surface of the gold layer on the cantilever was also studied as a function of the thickness and for a constant deposition rate of $0.02 \mathrm{~nm} / \mathrm{s}$. Figure 2 shows

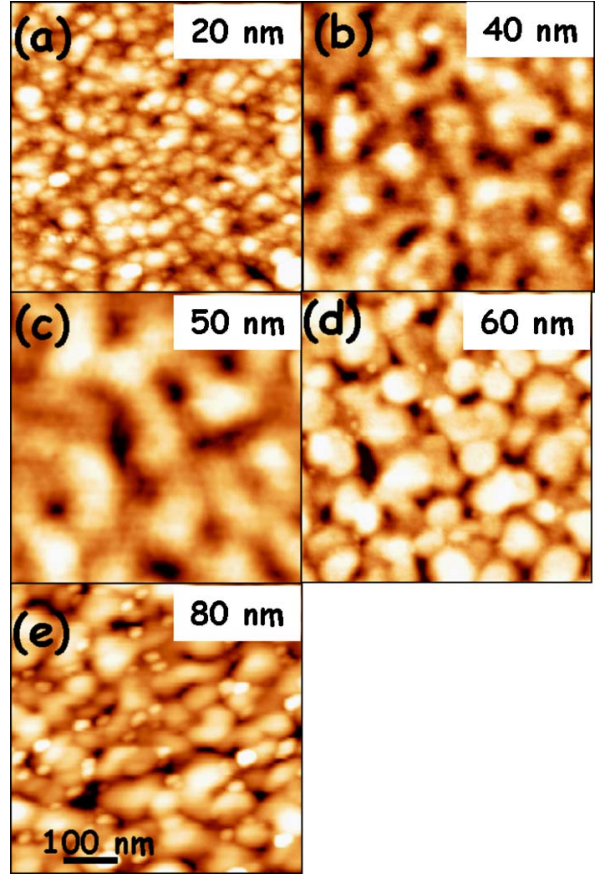

FIG. 2. (Color online) Atomic force microscopy topography images (0.5 $\times 0.5 \mu \mathrm{m}^{2}$ ) of gold films deposited on the cantilevers at $0.2 \AA / \mathrm{s}$ for different thicknesses: (a) $20 \mathrm{~nm}$, (b) $40 \mathrm{~nm}$, (c) $50 \mathrm{~nm}$, (d) $60 \mathrm{~nm}$, and (e) $80 \mathrm{~nm}$. An intermediate $2 \mathrm{~nm}$ thick chromium layer was deposited at $0.02 \mathrm{~nm} / \mathrm{s}$ to enhance the adhesion between the gold layer and the cantilever.

the AFM topographic images of the most representative thicknesses. The $20 \mathrm{~nm}$ thick gold film exhibits a granular surface, in which the grain boundaries are well defined. The grain size is $30-35 \mathrm{~nm}$ and the roughness is $1.7 \mathrm{~nm}$ [Fig. 2(a)]. When the thickness increases to $40 \mathrm{~nm}$, the grain size increases to 50-60 $\mathrm{nm}$ and the grain boundaries are less defined. This suggests the beginning of a coalescence process that results into a decrease of the roughness form 1.7 to $0.95 \mathrm{~nm}$ [Fig. 2(b)]. The coalescence process is more clearly distinguished in the $50 \mathrm{~nm}$ thick gold film [Fig. 2(c)]. The surface shows the formation of large islands of about $100 \mathrm{~nm}$ in size that are joined together through elongated gold necks. When the thickness is increased to $60 \mathrm{~nm}$, the surface structure changes abruptly, showing well-defined grains and sharp grain boundaries [Fig. 2(d)]. The grain size is about $60 \mathrm{~nm}$ and the roughness increases from 0.9 to $1.6 \mathrm{~nm}$. This morphology suggests the end of the coalescence process and the growth of more material on top the coalesced film observed for $50 \mathrm{~nm}$. Further deposition results into further increase of the roughness, whereas the size of the grains remains similar, as shown in the topography images of the $80 \mathrm{~nm}$ thick gold films [Fig. 2(d)]. This indicates that the growth is now mainly vertical to the surface with small lateral atom diffusion.

\section{B. Gold-film-induced cantilever strain}

The residual stress induced by the gold film deposited on the cantilevers was characterized by measuring the cantilever profile ${ }^{24,25}$ with subnanometer resolution by the scanning optical technique described in Sec. II. ${ }^{19}$ The measurements were performed in de-ionized water, as the present study is 


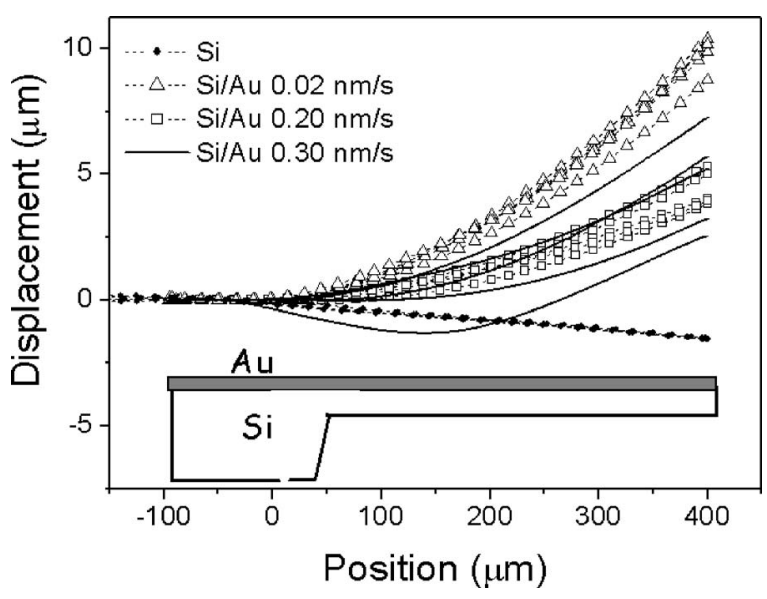

FIG. 3. Profiles of silicon cantilevers from the same array without gold coating (--) and coated with a $60 \mathrm{~nm}$ thick gold layer deposited at different rates: $0.02 \mathrm{~nm} / \mathrm{s}(-\triangle-), 0.2 \mathrm{nms}(\square /)$, and $0.3 \mathrm{~nm} / \mathrm{s}$ (line). A schematic depiction of the cantilever is also shown to relate the sign of the cantilever bending to the orientation of the bimetallic cantilever. The profiles were obtained in water.

oriented to study molecular adsorption in aqueous solutions. However, the relative variation between the profiles did not noticeably change in air. The profiles were measured in cantilever arrays in order to determine the reproducibility of the residual stress induced by the gold film.

Figure 3 shows the profiles of cantilever arrays coated with a $60 \mathrm{~nm}$ thick gold film deposited at 0.02, 0.2, and $0.3 \mathrm{~nm} / \mathrm{s}$. The profiles of the uncoated silicon cantilevers are also depicted. The uncoated silicon cantilevers exhibit similar profiles with a small deflection of $-1.5 \mu \mathrm{m}$ and a small deviation between the cantilevers in the array of $\pm 150 \mathrm{~nm}$. Hence, these cantilevers are a good reference to understand the effect of the gold coating and subsequent molecular adsorption on the surface stress. The gold coating induces an important cantilever bending towards the gold surface that strongly depends on the growth rate of the gold film. The sign of the cantilever bending is consistent with the buildup of tensile surface stress in the gold layer. The maximum induced cantilever bending is found when the gold is deposited at a rate of $0.02 \mathrm{~nm} / \mathrm{s}$. The mean deflection is $10.0 \mu \mathrm{m}$ with a small dispersion between cantilevers in the same array of $\pm 0.65 \mu \mathrm{m}$. For the gold film deposited at $0.2 \mathrm{~nm} / \mathrm{s}$, the deflection of the cantilevers decreases to a mean value of $4.40 \mu \mathrm{m}$ with a dispersion of $\pm 0.70 \mu \mathrm{m}$. When the deposition rate is increased to $0.3 \mathrm{~nm} / \mathrm{s}$, the mean bending of the cantilevers is similar to that found at $0.2 \mathrm{~nm} / \mathrm{s}, 4.80 \mu \mathrm{m}$; however, the dispersion between cantilevers dramatically increases to $\pm 1.90 \mu \mathrm{m}$.

The tensile stress induced by the gold coating can be related to the discontinuous nature of the gold film, as shown in the AFM pictures (Fig. 1). The maximum tensile stress observed at the deposition rate of $0.02 \mathrm{~nm} / \mathrm{s}$ is related to the process initiated when the growing islands contact each other and "zip" together to form a grain boundary. This process is driven by the reduction of the overall surface energy and imposes a cost of increased strain energy. ${ }^{26}$ This explanation is consistent with the well-defined grain boundaries observed in Fig. 1(a). For the higher deposition rates (0.2 and

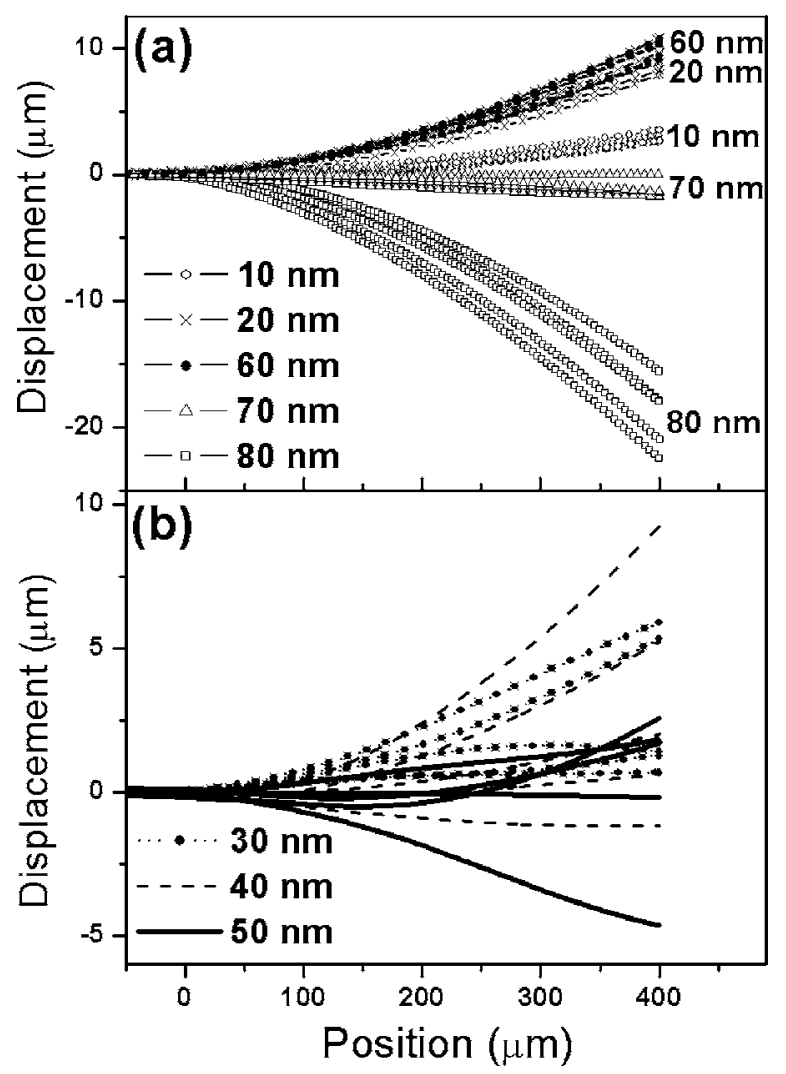

FIG. 4. Profile of silicon cantilevers from the same array coated with different thicknesses of gold at a constant deposition rate of $0.02 \mathrm{~nm} / \mathrm{s}$. (a) Profiles of the cantilevers before $(10$ and $20 \mathrm{~nm})$ and after the coalescence process of the gold layer $(60,70$, and $80 \mathrm{~nm})$. (b) Profiles of the cantilevers for which the gold film is at the coalescence stage $(30,40$, and $50 \mathrm{~nm})$. The profiles were measured in water.

$0.3 \mathrm{~nm} / \mathrm{s}$ ), the cantilever bending is smaller. This indicates that the cost of elastic energy in the nanoislands for a reduction of the interfacial energy is smaller. The AFM topographic images show less defined grain boundaries and the partial coalescence of the islands. This suggests that the reduction of the surface energy is also driven by adatom diffusion from the nanoislands into the grain boundaries. ${ }^{26,27}$ This process is favored by the liquidlike behavior of the surface atoms for small grains. For the gold film grown at $0.3 \mathrm{~nm} / \mathrm{s}$, whose surface topography clearly shows the coalescence of grains [Fig. 1(c)], there is a large dispersion in the profiles of the cantilevers, of about $80 \%$, and an uneven curvature along each cantilever. Thus, some cantilevers exhibit compressive stress (positive curvature) near the clamping that turns into tensile (negative curvature) towards the free beam end.

The dependence of the cantilever profile on the gold film thickness was also investigated for a deposition rate of $0.02 \mathrm{~nm} / \mathrm{s}$ (Fig. 4). Initially, for thicknesses of 10 and $20 \mathrm{~nm}$, the cantilevers bend towards the gold surface, indicating tensile stress. The maximum tensile stress is found for the $20 \mathrm{~nm}$ thick gold film, with a deflection of $9.10 \pm 0.70 \mu \mathrm{m}$ [Fig. 4(a)]. The large tensile stress is related to the initial stage of the gold film prior to the coalescence, in which atom diffusion is not produced, and the grain boundaries are formed at the expense of elastic energy. For thicknesses from 30 to $50 \mathrm{~nm}$, the situation is completely differ- 
ent [Fig. 4(b)]. The mean deflection decreases with the thickness from 2.90 to $0.30 \mu \mathrm{m}$. This indicates that an increasing relative compressive stress is developing at this stage. In addition, the dispersion in residual stress between the cantilevers dramatically increases, being of about $\pm 3-4 \mu \mathrm{m}$. In fact, there are arrays composed of cantilevers bent towards the gold and cantilevers bent towards the silicon side, which indicates that some cantilevers have overall compressive stress. Note that the corresponding AFM topographic images show the process of formation of elongated islands arising from fusion of previous smaller islands (Fig. 2). Again, the relative increase (decrease) of compressive (tensile) stress and the large dispersion in the cantilever deformation are related to the coalescence process via adatom migration. This allows the relief of the high strain energy accumulated in the $20 \mathrm{~nm}$ thick gold layer. When the gold film thickness is increased from 50 to $60 \mathrm{~nm}$, the behavior of the cantilevers is noticeably different. The cantilevers exhibit a maximum of tensile stress corresponding to a mean deflection of $10.0 \mu \mathrm{m}$. This is accompanied by a relative minimum in the deviation of the deflection between cantilevers, of about $12 \%$. This suggests that at this stage the stress is dominated by the grain boundaries formed on top of the coalesced $50 \mathrm{~nm}$ thick film (Fig. 2). At this stage the size of the gold islands is large enough to impede the stress relaxation via surface diffusion. In fact, the melting point of gold nanoparticles increases with their size. For gold films thicker than $60 \mathrm{~nm}$, there is an increasing relative change of the deflection towards the silicon surface, reaching a value of $-19.0 \pm 3.5 \mu \mathrm{m}$ for a thickness of $80 \mathrm{~nm}$. The increase of compressive stress during the postcoalescence process has been widely reported. ${ }^{28,29}$ It has been attributed to capillaryinduced growth stress due to the smaller lattice parameter of the nanoislands with respect to equilibrium. ${ }^{14}$ In addition, the effect of the increase in the surface chemical potential caused by the deposition of atoms from the vapor has been pointed out, which induces atoms to flow into the grain boundaries. ${ }^{30}$

An important result is that the coalescence process via atom diffusion is characterized by a lack of reproducibility in the resulting cantilever profile. This also reflects into a nonuniform curvature in each cantilever. In fact, some cantilevers show coexistence of regions with negative and positive curvatures. This suggests that the mechanism of gold diffusion can be very fast and unpredictable. ${ }^{31,32}$ A tentative explanation is the formation of a relatively unstable equilibrium state with a delicate and complex interplay between the nanoisland strain energy, the atom diffusion energy, the surface and grain boundary energies, and the mechanical energy stored in the cantilever.

\section{Effect of molecule adsorption on the cantilever profile}

To elucidate the effect of the gold layer nanostructure and residual stress on the sensitivity and reproducibility of microcantilever sensors, we have studied the response of the gold-coated cantilever arrays to the adsorption of $\mathrm{MCH}$. $\mathrm{MCH}$ forms a self-assembled monolayer on the gold-coated side of the cantilevers due to the strong bond between the thiol terminal group and the gold. ${ }^{10}$ The effect of molecular

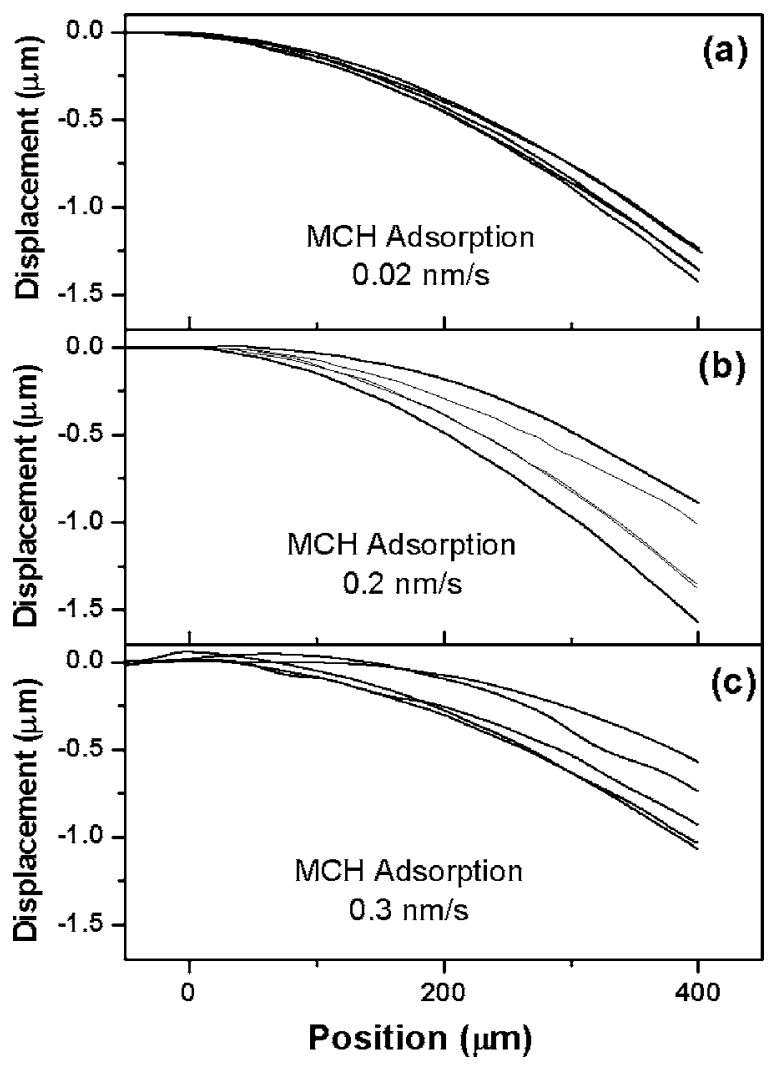

FIG. 5. Relative change of the deflection profile due to the $\mathrm{MCH}$ adsorption for arrays of five cantilevers coated with $60 \mathrm{~nm}$ of gold at deposition rates of (a) $0.02 \mathrm{~nm} / \mathrm{s}$, (b) $0.2 \mathrm{~nm} / \mathrm{s}$, and (c) $0.3 \mathrm{~nm} / \mathrm{s}$. The measurements were performed in water.

adsorption on the surface stress is discussed by measuring the difference between the profiles of the cantilever after and before molecular adsorption.

Figure 5 shows the change of the cantilever profile due to the $\mathrm{MCH}$ adsorption in water for the five cantilevers of an array with a $60 \mathrm{~nm}$ thick gold layer deposited at three different rates. The cantilevers bend towards the silicon side, indicating that $\mathrm{MCH}$ adsorption induces compressive surface stress on the gold surface. The amount of bending and its uniformity between different cantilevers depend largely on the deposition rate of the gold layer. Thus, for the cantilever arrays with the gold film deposited at a rate of $0.02 \mathrm{~nm} / \mathrm{s}$, the adsorption of $\mathrm{MCH}$ induces an average displacement of the cantilever end of $-1.3 \mu \mathrm{m}$ with a dispersion between cantilevers of $12 \%$ [Fig. 5(a)], whereas for the cantilevers gold coated at a rate of $0.2 \mathrm{~nm} / \mathrm{s}$, the average deflection is similar, $-1.2 \mu \mathrm{m}$, but the dispersion is of about $45 \%$ [Fig. $5(\mathrm{~b})]$. When the gold layer is deposited at a rate of $0.3 \mathrm{~nm} / \mathrm{s}$, the effect is more dramatic, and the average deflection falls to $-0.9 \mu \mathrm{m}$ and the dispersion is of $48 \%$ [Fig. 5(c)]. Hence, the higher the growth rate of the gold layer, the higher the deviation in the adsorption-induced bending between different cantilevers and the smaller the response. We recall that higher growth rates of the gold film produce a higher degree of coalescence, and smaller amount and higher deviation in residual tensile stress.

Figure 6 shows the relationship between the adsorptioninduced bending and the gold film thickness. For a major 

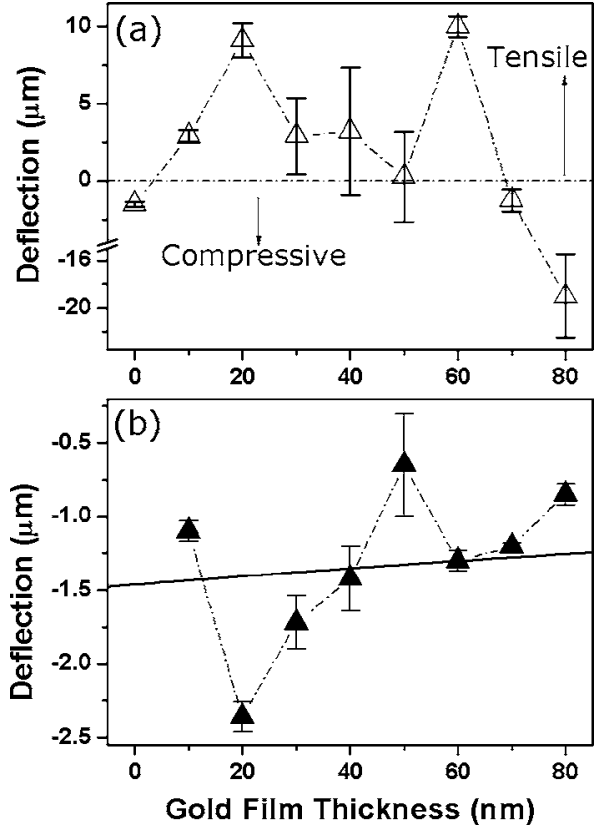

FIG. 6. (a) Cantilever free end deflection as a function of gold film thickness. (b) Relative displacement of the cantilever free end induced by the adsorption of $\mathrm{MCH}$ in water vs the gold layer thickness. The gold deposition rate was $0.02 \mathrm{~nm} / \mathrm{s}$. The continuous line in (b) represents the theoretical decrease in the cantilever response only due to the cantilever stiffening caused by the gold coating. Positive values correspond to tensile stress, whereas the negative values correspond to compressive stress.

understanding of the experimental data, we also show a graph of the residual deflection versus the gold film thickness [Fig. 6(a)]. The MCH adsorption always produces the cantilever bending towards the silicon side, indicating compressive stress. The amount of stress critically depends on the thickness of the gold film [Fig. 6(b)]. Two relative maxima of cantilever response to adsorption are found for the gold films prior and posterior to the coalescence process that are found for the thickness values of 20 and $60 \mathrm{~nm}$. The cantilever deflections are -2.4 and $-1.3 \mu \mathrm{m}$, respectively. In addition, these cantilevers provide the highest uniformity in the response to $\mathrm{MCH}$ adsorption with a dispersion of $8 \%-10 \%$. These gold films previously exhibited the highest amount of residual tensile stress and the lowest dispersion in the cantilever profiles [Fig. 6(a)]. In both films, the high interfacial energy is reduced by the formation of grain boundaries between the well-defined nanoislands. This process is mainly driven by tensile stress buildup in the islands. MCH adsorption passivates the gold surface, largely reducing the surface energy and alleviating the tensile residual stress. The minimum cantilever response and maximum dispersion are found for the $50 \mathrm{~nm}$ thick gold film, and are $0.60 \mu \mathrm{m}$ and $110 \%$, respectively. For this thickness, the gold film previously showed the highest degree of coalescence, and the cantilevers had a relative minimum of residual tensile stress with a high degree of dispersion. For thicknesses higher than $60 \mathrm{~nm}$, the MCH-induced cantilever response slowly decreases with the gold film thickness and the deviation between cantilevers is of about $17 \%$. For these thicknesses, we have found a relative increase of compressive residual stress with the thickness. The line in Fig. 6(b) depicts the theoretical deflection variation with the gold thickness if the cantilever response to the $\mathrm{MCH}$ would only depend on the mechanical properties. For a given value of surface stress, the cantilever deflection is inversely proportional to the thickness square [see Eq. (1)]. It can be seen that the increase of cantilever stiffness due to the gold film does not play a significant role in our experimental results.

\section{DISCUSSION}

The experimental results shown in Sec. III, grain size and roughness of the gold film, and values of the cantilever deflection after the gold coating and $\mathrm{MCH}$ adsorption are summarized on Table I. In addition, the surface stress induced by the gold coating and subsequent $\mathrm{MCH}$ adsorption are calculated by fitting the profiles with a second-order polynomial to determine the curvature and applying Stoney's equation [Eq. (1)]. We use a Young's modulus for silicon of $E=169 \mathrm{GPa}$ and a Poisson's coefficient of $v=0.064$. The surface stress induced by the gold layer ranges from -5 to $2.6 \mathrm{~N} / \mathrm{m}$, whereas the $\mathrm{MCH}$-induced surface stress goes from -0.05 to $-0.69 \mathrm{~N} / \mathrm{m}$. The values of surface stress correspond with the cantilever immersed in de-ionized water.

TABLE I. Experimental values of the grain size, roughness (root mean square) of the gold surface, surface stress induced by the gold film on the silicon cantilever, and surface stress induced by the $\mathrm{MCH}$ adsorption on the gold-coated cantilever as a function of the thickness and deposition rate of the gold film. The surface roughness was determined by calculating the root mean square (rms) of the AFM images for areas of $1 \times 1 \mu \mathrm{m}^{2}$.

\begin{tabular}{cccccccccccc}
\hline \hline Thickness (nm) & 0 & 10 & 20 & 30 & 40 & 50 & 60 & 60 & 60 & 70 & 80 \\
\hline $\begin{array}{c}\text { Deposition rate } \\
\text { (nm/s) }\end{array}$ & 0.02 & 0.02 & 0.02 & 0.02 & 0.02 & 0.02 & 0.02 & 0.2 & 0.3 & 0.02 & 0.02 \\
Grain size (nm) & & & & & & & & & & & \\
Roughness (nm) & 0.3 & 2.6 & $30-35$ & $40-50$ & $50-60$ & $90-110$ & $50-70$ & $30-70$ & $60-80$ & $60-80$ & $90-100$ \\
Au & $\ldots$ & 1.81 & 2.29 & 0.60 & 1.33 & 0.7 & 2.58 & 1.47 & 2.11 & -0.10 & -5.04 \\
surface stress & & \pm 0.22 & \pm 0.39 & \pm 0.09 & \pm 1.1 & \pm 1.3 & \pm 0.16 & \pm 0.35 & \pm 0.66 & \pm 0.33 & \pm 0.44 \\
(N/m) & & & & & & & & & & & \\
MCH surface & $\ldots$ & -0.398 & -0.687 & -0.053 & -0.414 & -0.185 & -0.377 & -0.452 & -0.09 & -0.252 & -0.277 \\
stress (N/m) & & \pm 0.041 & \pm 0.041 & \pm 0.008 & \pm 0.037 & \pm 0.023 & \pm 0.023 & \pm 0.039 & \pm 0.11 & \pm 0.034 & \pm 0.065 \\
Au & -1.50 & 2.90 & 9.10 & 2.90 & 3.20 & 0.3 & 10.00 & 4.40 & 4.80 & -1.30 & -19.00 \\
deflection ( $\mu \mathrm{m})$ & \pm 0.15 & \pm 0.35 & \pm 0.70 & \pm 2.5 & \pm 4.10 & \pm 2.5 & \pm 0.65 & \pm 0.70 & \pm 1.90 & \pm 0.75 & \pm 0.35 \\
MCH & - & -1.10 & -2.36 & -1.72 & -1.42 & -0.65 & -1.30 & -1.24 & -0.87 & -1.20 & -0.86 \\
deflection $(\mu \mathrm{m})$ & & \pm 0.07 & \pm 0.10 & \pm 0.18 & \pm 0.22 & \pm 0.35 & \pm 0.07 & \pm 0.28 & \pm 0.21 & \pm 0.10 & \pm 0.07 \\
\hline \hline
\end{tabular}


The performance of cantilever sensors critically depends on two parameters, the cantilever sensitivity and the reproducibility of the response between different cantilevers. The results shown above imply that the uniformity of the response of the cantilevers to molecular adsorption is optimized by working with cantilevers with low deviation in the residual stress. This is found in cantilevers in which the gold coating has not undergone coalescence or in the first stages after coalescence. These gold films are characterized by large values of tensile stress of about $2.3-2.6 \mathrm{~N} / \mathrm{m}$. Contrarily, the reproducibility of the cantilever response to molecular adsorption is very poor when the gold coating is at the coalescence stage. These films produce small residual tensile stress in the cantilevers and large deviation in the residual bending.

The relationship between the amount of cantilever response to molecular adsorption and the gold film properties is not clear. Two important mechanisms related to the morphology of the gold surface have been reported: the nanostructure of the gold film and the size of the grains. A higher nanostructuring of the gold surface provides an increased effective area and therefore a major number of binding sites. ${ }^{15}$ In addition, it produces confinement of the molecules in nanocavities, increasing the intermolecular forces such as the solvation, steric, osmotic, and hydration forces with respect to flat surfaces. On the other hand, Godin et al. ${ }^{16}$ found that the grain size of the gold film strongly influences both the kinetics of the formation of self-assembled monolayers and the final monolayer structure. In their study, for grain sizes smaller than $100 \mathrm{~nm}$, the monolayer cannot achieve the highly ordered standing-up phase and the final surface stress in air was of about $0.5 \mathrm{~N} / \mathrm{m}$. The standing-up phase was found for large grains $(>500 \mathrm{~nm})$ providing a surface stress of about 30 times higher.

Since the gold films studied here exhibited grain sizes smaller than $100 \mathrm{~nm}$, we can assume that most of the $\mathrm{MCH}$ molecules are lying down on the gold and the grain size is not determinant in the adsorption-induced surface stress as can be observed in Table I. On the other hand, we have not found a ready relationship between the adsorption surface stress and the roughness of the gold film (Table I). This implies that the gold nanostructure does not play a fundamental role in the surface stress induced by $\mathrm{MCH}$ adsorption. We have found that in our conditions, the cantilever sensitivity to molecular adsorption primarily depends on the residual surface stress of the gold film that is intimately connected to the phenomena of grain boundary formation and coalescence that minimizes the effect of the large gold surface energy. The higher values of adsorption-induced surface stress are found for the gold films with the larger values of tensile stress. In these films, there is a high elastic strain energy accumulated in the nanoislands that is partly relieved by the attachment of the $\mathrm{MCH}$ molecules. The $\mathrm{MCH}$ adsorption decreases the gold surface energy and therefore the elastic energy accumulated in the grains.

\section{CONCLUSIONS}

We have studied the role of the gold film deposited on microcantilevers on the sensitivity and reproducibility of their static response to molecular adsorption. Different morphologies of the gold film were obtained by depositing gold layers with several thicknesses (from 10 to $80 \mathrm{~nm}$ ) at a deposition rate of $0.02 \mathrm{~nm} / \mathrm{s}$ and depositing a $60 \mathrm{~nm}$ thick layer at different deposition rates $(0.02,0.2$, and $0.3 \mathrm{~nm} / \mathrm{s})$. The residual stress induced by the gold layer strongly depends on the growth conditions and it is related to the nanostructure of the gold surface. At low deposition rates and small thicknesses, the gold film has not undergone coalescence and the surface is formed by well-defined grains and well-delineated grain boundaries. These gold layers have large tensile stress, indicating that the grain boundaries are formed at the expense of strain energy in the grains. These cantilevers exhibit the highest sensitivity and reproducibility to molecular adsorption. However, for larger thicknesses and higher deposition rates, coalescence of gold nanoislands occurs due to the "cold melting" phenomena produced in the small gold nanoparticles. The coalescence results into a large relative decrease (increase) of the tensile (compressive) stress. In addition, the coalescence process produces a significant dispersion in the cantilever residual stress and curvature. The cantilevers coated with a coalesced gold layer present a smaller sensitivity and a low reproducibility in measurements of molecular adsorption. We conclude that control of the process of gold coating on cantilevers is critical for the reliability of micromechanical sensors. Therefore the gold surface nanostructure and induced residual stress should be first examined before application of the cantilevers for chemical and biological detection.

\section{ACKNOWLEDGMENTS}

This work was supported by the Spanish Ministry of Science (GEN2001-4856-C13-11) and CSIC (200550M056). We acknowledge A. Tarín for his help with the AFM images.

${ }^{1}$ N. V. Lavrik, M. J. Sepaniak, and P. G. Datskos, Rev. Sci. Instrum. 75, 2229 (2004)

${ }^{2}$ H. P. Lang, M. Hegner, and C. Gerber, Mater. Today 8, 30 (2005).

${ }^{3}$ R. Raiteri, M. Grattarola, H. J. Butt, and P. Skládal, Sens. Actuators B 79, 115 (2001)

${ }^{4}$ H. Ibach, J. Vac. Sci. Technol. A 12, 2240 (1994).

${ }^{5}$ W. Haiss, Rep. Prog. Phys. 64, 591 (2001).

${ }^{6}$ Z. Hu, T. Thundat, and R. J. Warmack, J. Appl. Phys. 90, 427 (2001).

${ }^{7}$ G. Wu, R. H. Datar, K. M. Hansen, T. Thundat, R. J. Cote, and A. Majumdar, Nat. Biotechnol. 19, 856 (2001).

${ }^{8}$ P. A. Rasmussen, A. V. Grigorov, and A. Boisen, J. Micromech. Microeng. 15, 1088 (2005).

${ }^{9}$ R. McKendry et al., Proc. Natl. Acad. Sci. U.S.A. 99, 9783 (2002).

${ }^{10}$ M. Álvarez, L. G. Carracosa, M. Moreno, A. Calle, A. Zaballos, L. M. Lechuga, A. C. Martínez, and J. Tamayo, Langmuir 20, 9663 (2004).

${ }^{11}$ M. Álvarez, A. Calle, J. Tamayo, A. Abad, A. Montoya, and L. M. Lechuga, Biosens. Bioelectron. 18, 649 (2003).

${ }^{12}$ N. A. Alcantar, C. Park, J. Pan, and J. N. Israelachvili, Acta Mater. 51, 31 (2003).

${ }^{13}$ S. Arcidiacono, N. R. Bieri, D. Poulikakos, and C. P. Grigoropoulos, Int. J. Multiphase Flow 30, 979 (2004).

${ }^{14}$ S. Iijima and P. M. Ajayan, J. Appl. Phys. 70, 5138 (1991).

${ }^{15}$ J. J. Headrick, M. J. Sepaniak, N. V. Lavrik, and P. G. Datskos, Ultramicroscopy 97, 417 (2003).

${ }^{16}$ M. Godin, P. J. Williams, V. Tabard-Cossa, O. Laroche, L. Y. Beaulieu, R. B. Lennox, and P. Grütter, Langmuir 20, 17 (2004).

${ }^{17}$ R. J. Koch, J. Phys.: Condens. Matter 6, 9519 (1994).

${ }^{18}$ J. Mertens, M. Álvarez, and J. Tamayo, Appl. Phys. Lett. 87, 234102 (2005).

${ }^{19}$ M. Alvarez and J. Tamayo, Sens. Actuators B 106, 687 (2005). 
${ }^{20}$ D. C. Miller, C. F. Herrmann, H. J. Maier, S. M. George, C. R. Stoldt, and K. Gall, Scr. Mater. 52, 873 (2005).

${ }^{21}$ K. L. Chopra and M. R. Randlett, J. Appl. Phys. 39, 1874 (1968).

${ }^{22}$ T. Pienkos, A. Proszynski, D. Chocyk, L. Gladyszewski, and G. Gladyszewski, Microelectron. Eng. 70, 442 (2003).

${ }^{23}$ M. C. Barnes, I. Jeon, D. Kim, and N. M. Hwang, J. Cryst. Growth 242, 445 (2002).

${ }^{24}$ S. Jeon and T. Thundat, Appl. Phys. Lett. 85, 1083 (2005).

${ }^{25}$ M. Helm, J. J. Servant, F. Saurenbach, and R. Berger, Appl. Phys. Lett. 87, 064101 (2005).

${ }^{26}$ J. A. Floro, S. J. Hearne, J. A. Hunter, P. Kotula, E. Chason, S. C. Seel, and C. V. Thompson, J. Appl. Phys. 89, 4886 (2001).
${ }^{27}$ M. José-Yacamán, C. Gutierrez-Wing, M. Miki, D.-Q. Yang, K. N. Piyakis, and E. Sacher, J. Phys. Chem. B 109, 9703 (2005).

${ }^{28}$ M. Pletea, W. Brückner, H. Wendrock, and R. Kaltofen, J. Appl. Phys. 97, 1 (2005).

${ }^{29}$ B. W. Sheldon, K. H. A. Lau, and A. Rajamani, J. Appl. Phys. 90, 5097 (2001).

${ }^{30}$ E. Chason, B. W. Sheldon, L. B. Freund, J. A. Floro, and S. J. Hearne, Phys. Rev. Lett. 88, 156103 (2002).

${ }^{31}$ C. Chung, T. Lin, J. Duh, and M. Tsai, Surf. Coat. Technol. 200, 4825 (2006).

${ }^{32}$ J. Proost and F. Spaepen, J. Appl. Phys. 91, 204 (2002). 